\title{
TENTATIVE COURSE OUTLINE
}

(THE COURSE WILL FOLLOW TO SOME DEGREE AN OUTLINE OF ARISTOTLEIAN RHETORIC)

1st Week

Overview of the course

Benjamin, Part 1,"Concepts of Communication", 1-9

2nd Week

Aristotleian Rhetoric

3rd Week

Invention

Ethos (Credibility)

Subject Selection (prior experience)

Benjamin, page 158

4th Week

Invention (continued)

Pathos (Emotional Appeals)

Maslow

Links to Familiar and Commonplaces

Benjamin, page 67 and page 157

5 th Week

Invention

Logos (Logical Appeals)

Thesis Formulation

Critical Thinking (Arnold Wood)

Benjamin, 153-157

6 th Week

Disposition

Outine and Content

Benjamin, 136-148

7 th Week

Style

Analogies

Vocabulary

Benjamin, 35-44

8th Week

Memory and Delivery

Verbal--Oral Interpretation and Enunciation

Visualization "Be Prepared to Speak"

Benjamin, 127-135 
9th Week

$$
\begin{aligned}
& \text { Memory and Delivery } \\
& \text { Nonverbal } \\
& \text { Benjamin, 45-56 }
\end{aligned}
$$

10 th Week

Listening

Benjamin, 24-34

11th Week

Group Processes

Leadership

Group Discussion

Benjamin, 87-99 and 100-115 